\title{
Variable Window Length Applied To A Modified Hanning Filter For Optimal Amplitude Estimation Of Power Systems Signals
}

\author{
Thiago R. F. Mendonça, Carlos H. N. Martins, Milena F. Pinto, Carlos A. Duque, IEEE Senior Member. \\ Electrical Engineering Department \\ Federal University of Juiz de Fora \\ Juiz de Fora, Brazil \\ thiago.mendonca@engenharia.ufjf.br
}

\begin{abstract}
Parameters estimation of electrical power system is relevant for monitoring, protection and control. Several methods are used to this purpose, such as Discrete Fourier Transform (DFT), Moving Average Filter (MAF), Hanning window, among others. However, some of these techniques require a number of samples within a complete number of cycles of the fundamental component in order to obtain better results. The oscillation of the system's frequency hinders the selection of a fixed window length. In this sense, in this paper is proposed a variable window length applied in a modified Hanning filter in order to optimally estimate the amplitude as well as eliminate harmonics and inter-harmonics of the power systems signals. The modified version of the Hanning filter proposed in this work enhances the magnitude response by altering the poles locations of the transfer function, achieving optimal removal of the first harmonic considering only one cycle of the signal which is unachievable by the conventional Hanning window, and the change of the window length according to the system's estimated frequency applied in this modified filter, allows for optimal removal of harmonics even when considering variable frequency.
\end{abstract}

Index Terms - Amplitude estimation, Interharmonics and Harmonics Attenuation, Modified Hanning Filter, Variable window length.

\section{INTRODUCTION}

The estimation of important parameters such as amplitude, phase and frequency from the electrical power system's signals is relevant for monitoring, protection and control purposes. Frequency estimation of fundamental component from the grid is crucial to enhance the estimation of other parameters, improving the monitoring capability and estimation reliability. The power quality monitoring has been a central subject of research and studies due to its relevance for industries. There are some international standards regarding the assessment of power quality, such as the IEC 61000 [1], which recommends certain degree of accuracy and methodology for monitoring and event detection (voltage dips, swell, flickers and spectral analysis).
Several methods are used to extract these parameters from the electric system, such as the Discrete Fourier Transform (DFT), Moving Average Filter (MAF), among others. Concerning the amplitude estimation, there are a considerable number of algorithm such as the least squares and weighted least squares methods[2], it is also possible to rely on the filterbank approach such as the recursive DFT, moving average and Hanning filters. In this paper is proposed a modification on the design of a Hanning filter in which the removal of the first harmonic is possible even when considering a single cycle, preserving its characteristics of interharmonic attenuation and consequently enhancing its applications for online power systems monitoring.

Usually these algorithms require an integer number of cycles of the fundamental component in order to obtain better results [3]. This is a straightforward procedure when the signal has a constant fundamental frequency. In that case, it is only necessary to define a fixed clock for the sampling rate, which will fit a predetermined number of samples per cycle. However, the fundamental frequency in an electrical power system signal is not constant; it can vary in few tenths of Hertz, reflecting the balance between generation and consumption of electrical energy or even higher variation during disturbances. According to the IEC 61000-4-30 [1], the permissible tolerance is $\pm 0.5 \mathrm{~Hz}$. In that case, the predetermined sample rate, chosen as if the fundamental was $60 \mathrm{~Hz}$, will not allow having a complete cycle, resulting in errors and jeopardizing the estimation of other parameters.

There are some techniques found in literature used to define a variable sampling period [4] or a variable window length [5] in order to ensure that the amount of samples contain an integer number of cycles. There is another approach based on resampling of the sampled signal that can be performed by software [6]. Although these enhancements present good results, it also brings other concerns and difficulties, i.e. storage of the variable sample period signal for offline analysis imposed by the variable sampling rate or error inserted by fractional window lengths, among others. 
Several papers were published regarding the usage of Hanning Window for harmonic analysis, for instance, in [7] it is discussed the application for harmonic decomposition based on recursive Hanning window. The effect of the Hanning window on the processing of harmonics and interharmonics in the IEC standard is referred in [8]. In this way, this work aims the application of a variable window length in a modified Hanning filter proposed by the authors in order to optimally estimate the amplitude as well as remove harmonics and interharmonics of a power systems signal, even when the frequency deviates from the initial fundamental value assumed.

This paper is organized as follows. The second section discusses in more details the variable window length method, describing implementation issues as well as difficulties imposed by its usage. Section three focuses on the variable window length method applied in a modified Hanning filter, describing how to perform the enhancement in the magnitude response of the Hanning filter. The fourth section of this work shows the simulation results obtained in the estimation of the amplitude of a signal with variable frequency and amplitude. Finally, in the last section is presented the conclusion and suggestions for future works.

\section{VARIABLE WINDOW LENGTH METHOD}

Whenever the observable window does not contain an integer number of cycles, it is defined in literature as an asynchronous sampling. This condition results in error when some algorithms, such as the DFT, are utilized. For this reason, the signal used by the algorithm should contain one or more complete cycles [3]. Some methods are proposed as a form of enhancing the leakage effect due to the asynchronous sampling [9]-[12].

Other form to enhance these effects is by avoiding the asynchronous sampling. This section discusses the variable window length technique utilized for this purpose. This procedure is referred in detail in [5] and [13].

Usually the number of samples considered in estimation algorithms is chosen based on the system frequency known a priori in order to fit in a complete cycle and avoid errors. However when the frequency changes, the predetermined number of samples utilized will not cover an integer number of cycles, so it is necessary to change the length of the observable window. This method consists in changing the size of the observable data in the algorithm based on the frequency estimation. The optimal window length to contain the predetermined number of samples per cycle depends on the actual frequency estimation and the sampling interval, as dictated by (1).

$$
\mathrm{L}=\frac{1}{T_{S} \cdot f}
$$

Where $T_{\mathrm{s}}$ is the sampling interval and $\mathrm{f}$ is the fundamental frequency of the signal. For each frequency estimator output, a new size of window is defined. However, it may be needed to vary in a fractional number depending on the value of the actual frequency. This fractional sized window results in error when it is used in the transformation algorithm. It is noteworthy that is possible to fix the fractional sized window issue by interpolation.

For demonstration, consider a case in which the sampling rate is calculated to fit 16 samples per cycle of a fundamental frequency of $60 \mathrm{~Hz}$ (i.e. Fs $=16 \times 60$ ). However, the actual frequency is $64 \mathrm{~Hz}$ (a huge deviation only for sake of illustration), thus with the sampling rate of 960 samples per second, 16 samples will cover more than a complete cycle. By applying (1), the correct window length should be 15 samples per cycle to cover a complete cycle of the $64 \mathrm{~Hz}$ fundamental frequency and avoid leakage effect in the transformation algorithm. It can be noted in Figure 1 that using the incorrect window length, the two observable data represented by the black window are different from each other.

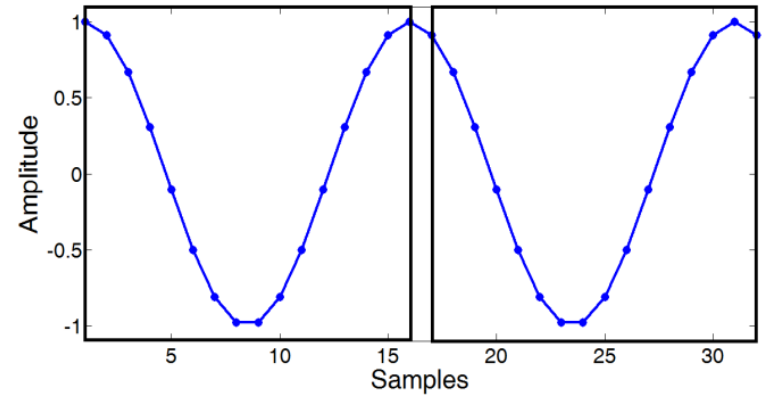

Figure 1. Incorrect window length for a $64 \mathrm{~Hz}$ signal.

In Figure 2 is shown the effect in the frequency domain of the incorrect window length. It is considered two cycles for the DFT algorithm, thus the frequency resolution given by (2) is $30 \mathrm{~Hz}$.

$$
\Delta \mathrm{f}=\frac{f}{N c}
$$

Where again $\mathrm{f}$ is the fundamental frequency and $\mathrm{Nc}$ is the number of cycles used by the transformation algorithm.

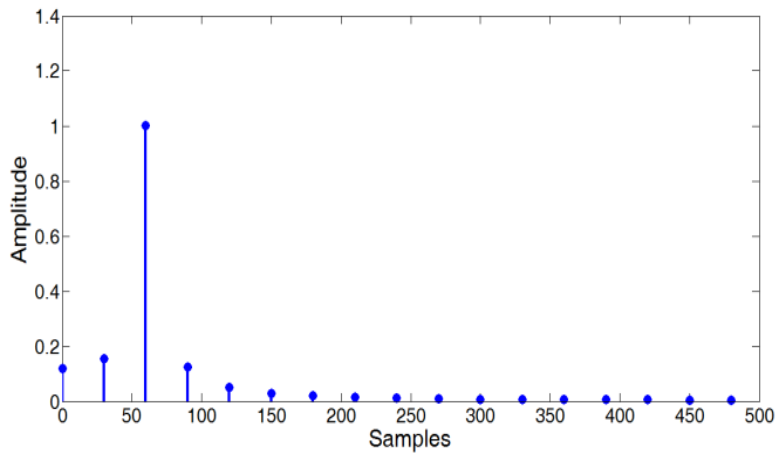

Figure 2. Leakage effect due to the incorrect window length.

The technique presented in this paper considers a new window length for every frequency estimate obtained by the frequency tracking algorithm (e.g. Phase Locked Loop, ZeroCrossing, Frequency Locked Loop, among others) updating its value according to (1). The main advantage of this method over the variable sampling rate is due to the fact that it is more convenient for data recording and analysis of an equally spaced samples vector. 


\section{VARIABLE WINDOW LENGTH APPLIED TO A MODIFIED HANNING FILTER}

The Hanning filter is much similar to a moving average filter (MAF). Nevertheless, it has the improvement in its transfer function that attenuates more rapidly than the latter, removing with more efficiency the interharmonic components. The transfer function of the moving average filter is described in (3). A key-point in utilizing this kind of filters is due to the elimination of harmonics.

$$
H_{M A F}(z)=\frac{1}{L} \cdot \frac{1-Z^{-L}}{1-Z^{-1}}
$$

The Hanning filter coefficients are defined according to (4) and its magnitude response is shown in Figure 3 along with the magnitude response of the MAF. Advantage of the Hanning filter is that besides removing harmonic components, it also attenuates with more efficiency the interharmonic components, since its lobe decays faster than the simple MAF. However, the first harmonic is not removed with the same efficiency.

$$
\mathrm{W}_{\text {Hann }}(\mathrm{n})=\frac{1}{2}\left(1-\cos \left(\frac{2 \cdot \pi \cdot n}{L}\right)\right)
$$

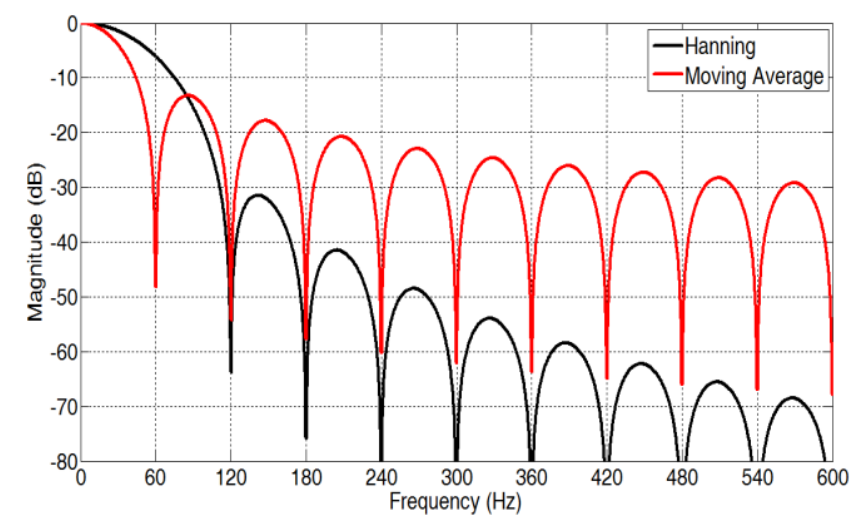

Figure 3. Magnitude response of a moving average and a Hanning filter considering a window length of 16 samples per cycle of a $60 \mathrm{~Hz}$ fundamental frequency.

The proposed modification of the Hanning filter aims to improve the magnitude response in such a way to attenuate the first harmonic as efficient as the MAF. By altering the poles location of the Hanning filter, it is possible to force the attenuation for the first harmonic as well. By bringing the poles of the first harmonic inside the unity circle, no more cancelation with the zeros occurs, and at each harmonic, the magnitude decreases. The poles and zeros in the $\mathrm{z}$ plane from the conventional Hanning filter and the modified version proposed in this paper is shown in Figure 4.

This modified Hanning filter has the magnitude response shown in Figure 5. It is noteworthy that the purpose to remove the first harmonic is achieved keeping the Hanning characteristic of interharmonic removal.

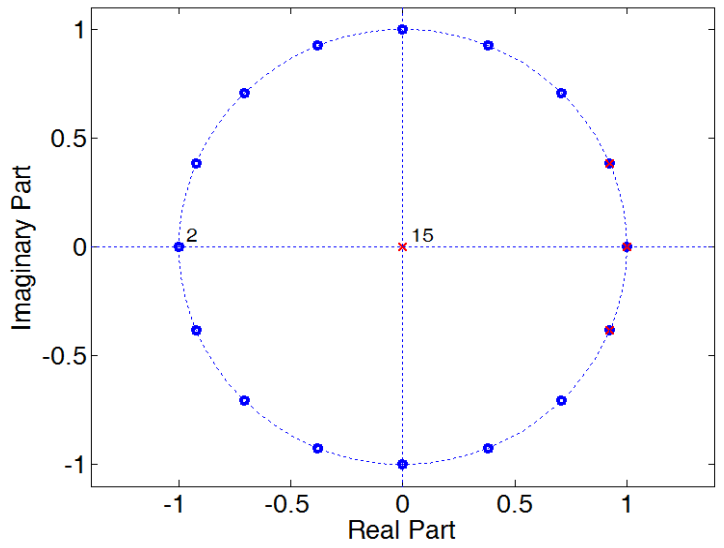

(a)

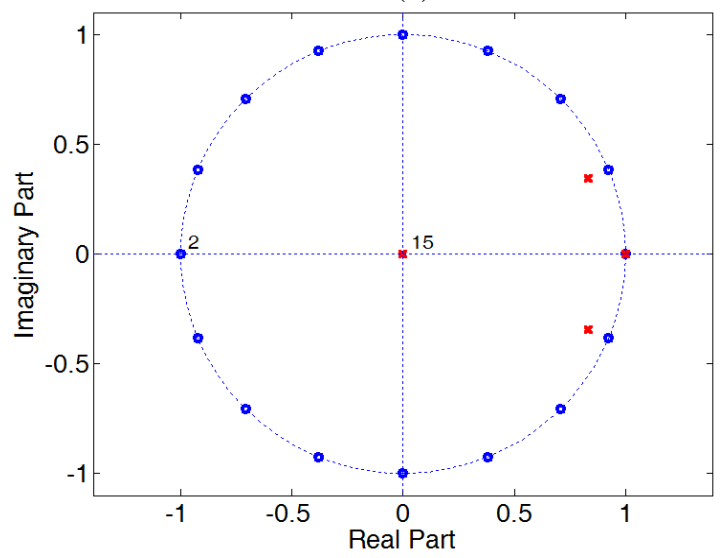

(b)

Figure 4. Poles and zeros representation in the z plane. (a) Hanning filter. (b) Modified Hanning filter

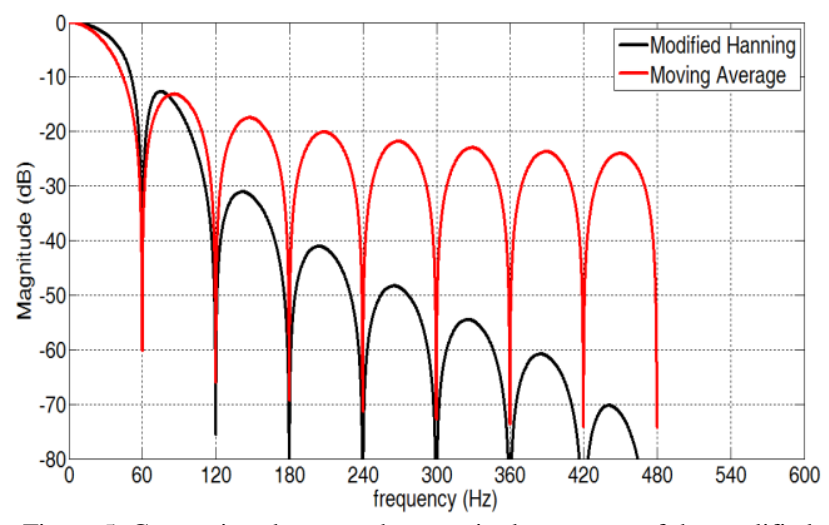

Figure 5. Comparison between the magnitude response of the modified Hanning filter with the MAF.

The effect of the selected window length is directly related with the cutoff frequency. In that way, the filter coefficients are optimally defined for a specific frequency, and will efficiently remove its harmonics components. However, if the fundamental frequency of the system varies, since the filter coefficients remain, so will its frequency response, in other words, it will keep considering the fundamental frequency as $60 \mathrm{~Hz}$, and optimally attenuating 
multiples of $60 \mathrm{~Hz}$. Within this context, it is proposed to apply the variable window length method in order to modify the coefficients and consequently optimally removes harmonics, as shown in Figure 6. The complete diagram of the proposed method for optimal amplitude estimation by means of the modified Hanning filter and the variable window length is presented in Figure 7.

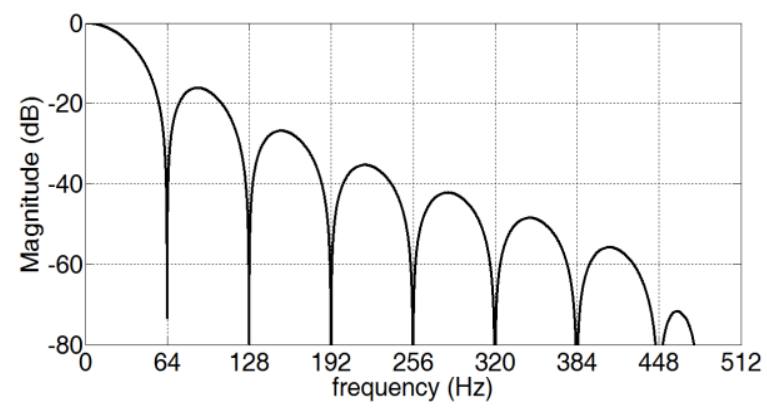

Figure 6. Magnitude response of the modified Hanning filter with its coefficients adapted for a $64 \mathrm{~Hz}$ fundamental component.

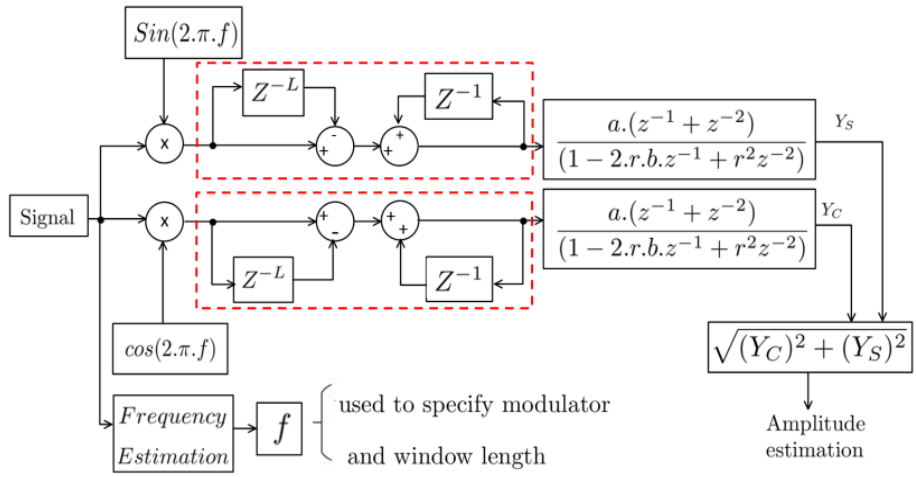

Figure 7. Diagram of the proposed method.

As can be seen in the frequency response, only the DC component of the signal is maintained. In this sense, for power systems application, it is necessary to modulate the signal in order to shift the fundamental frequency to the pass band of the proposed filter. The output of the frequency estimator is used to change the modulator and to modify the window length as the fundamental frequency of the system changes. The recursive implementation of (3) is depicted at the diagram as a traced red block. The block that follows regards the modified Hanning filter. L is the window length, the coefficient $a$ is calculated as $1-\cos (\theta)$ and $b$ is $\cos (\theta)$, where $\theta$ is $2 \pi / L$. The coefficient $r$ is the pole location in the $\mathrm{z}$ transformation plane, therefore has to be chosen as a value lower than unity, comprising the proposed modification. Note that in the diagram that the modulation is performed by the sine and cosine functions in order to obtain in phase and quadrature components $Y c$ and $Y s$. The output of both structures is conveniently combined to generate the amplitude and phase of the signal.

\section{Simulation Results}

The proposed method is capable of estimating the amplitude of a sinusoidal signal even with a variable frequency, adjusting its parameters accordingly and achieving good results. In this section, the results and comparisons between the modified Hanning filter with and without the variable window length are presented.

To validate the amplitude estimator under frequency variation, the algorithm is applied to a $60 \mathrm{~Hz}$ sinusoidal signal with abrupt change to $64 \mathrm{~Hz}$. Another test signal generated in order to validate the proposed method is an abrupt change in amplitude. Since the test signal is not corrupted by noise, a simple zero-crossing algorithm is used to track the frequency change.

In Figure 8, the amplitude estimation using conventional windowing method is presented. It can be noted that after the variation in frequency, the length of the window utilized by the algorithm has lower accuracy, presenting considerable oscillation in its value as showed in Figure 9.
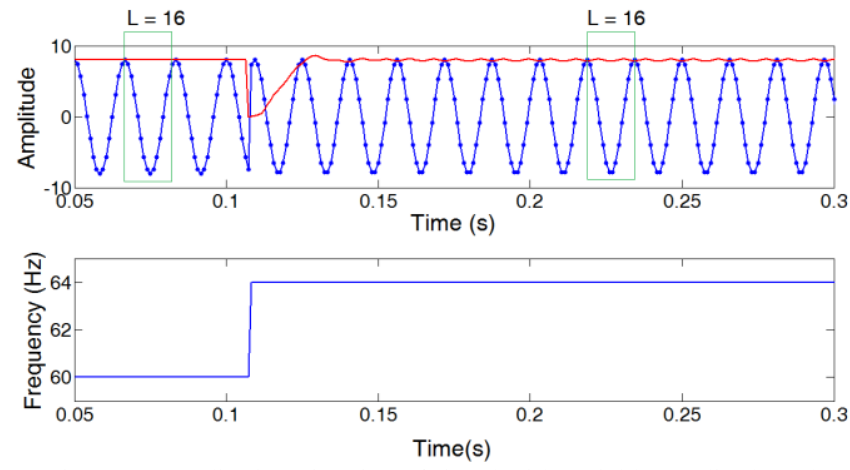

Figure 8. Amplitude estimation of signal with variable amplitude and frequency without the proposed method.

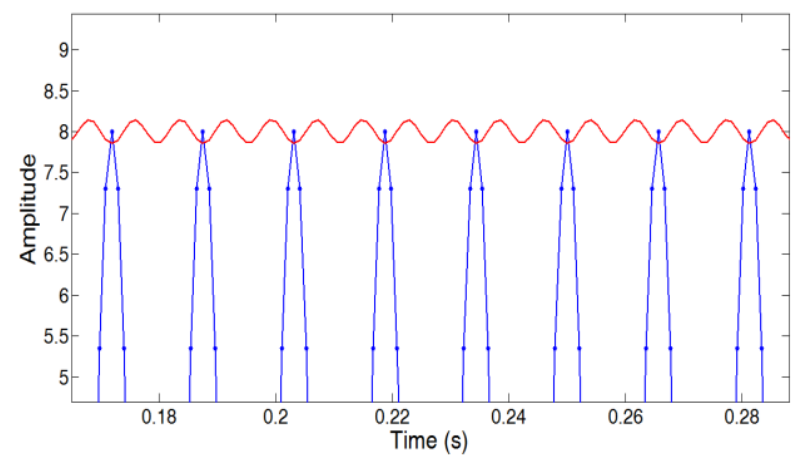

Figure 9. Oscilation in the amplitude estimation due to the badly chosen window length.

By using the frequency information provided by the estimator to change the window size, better results are obtained. Figure 10 shows the amplitude estimation for the same signal, considering the variable window length. Note that it shows better precision obtained by the correct window length for the new measured frequency. It is important to highlight that in both tests, the modified Hanning filter is used. However, in the latter the variable window length technique is applied. 

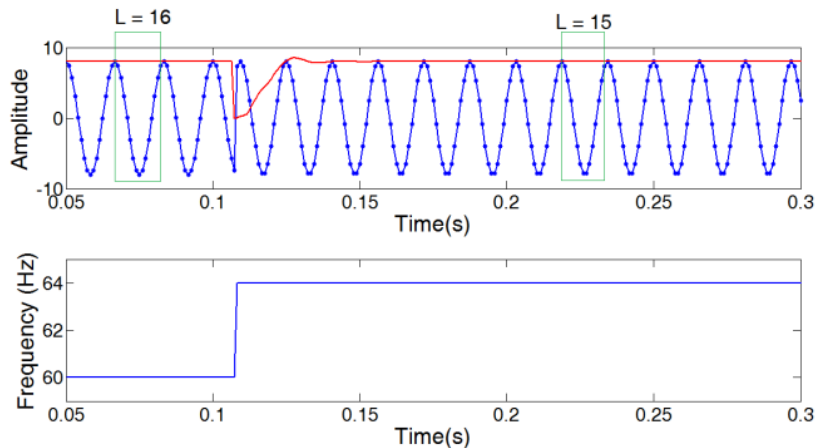

Figure 10. Amplitude estimation of signal with variable amplitude and frequency using the proposed method.

In Figure 11, the results obtained for tracking amplitude with abrupt change is presented. For this case, where no frequency variation exists, the conventional windowing method obtains similar results.

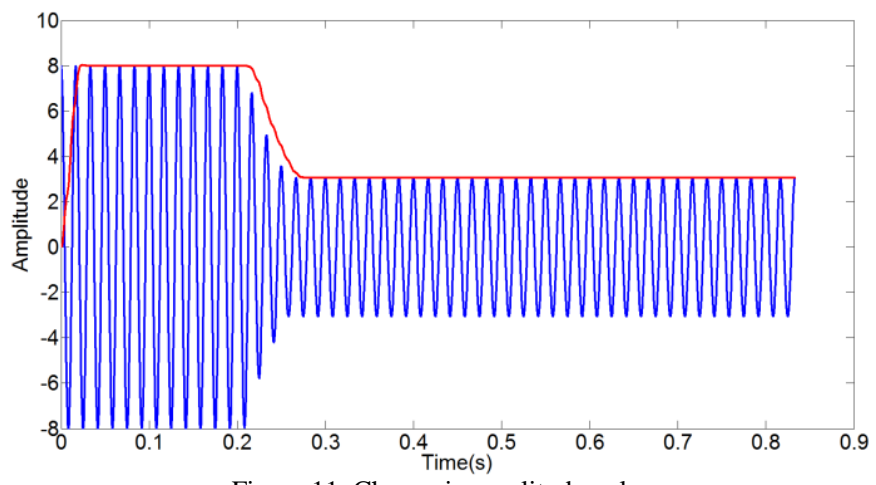

Figure 11. Change in amplitude only.

The modified approach is capable of estimating the amplitude even when in presence of harmonics and interharmonics. In Figure 12 it is shown the performance of the proposed method estimating the amplitude of a $60 \mathrm{~Hz}$ fundamental frequency corrupted by the second and fifth harmonic with $25 \%$ and $50 \%$ amplitude respectively and an interharmonic of $318 \mathrm{~Hz}$ with $12,5 \%$ amplitude.
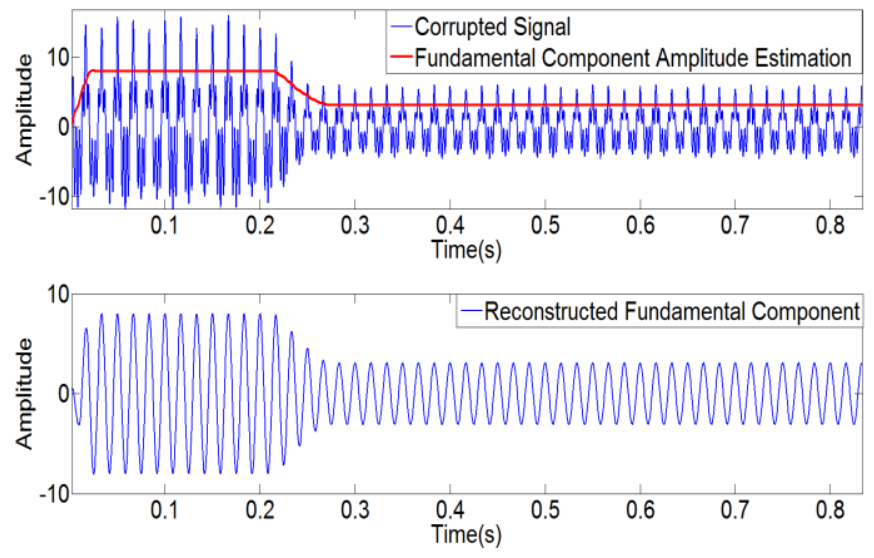

Figure 12. Amplitude estimation of a corrupted signal.

\section{CONCLUSION}

This work has shown a brief review concerning the variable window length method, highlighting its advantages and drawbacks. Additionally, it has presented a modification in Hanning window that allows the harmonic removal, including the first harmonic, considering only one cycle. It has performed a comparison between the magnitude response of the modified Hanning window with MAF in order to show its good performance of inter-harmonic removal.

Moreover it has discussed its usage for amplitude estimation even in presence of harmonics and interharmonics components. These results were performed varying both amplitude and frequency. As can be seen, the combination of the modified Hanning filter with the variable window length has tracked the amplitude with accuracy. As expected, the results have shown better performance for amplitude estimation comparing with a conventional Hanning window.

For future works, it is intended to develop the algorithm for interpolation of window length in case of fractional size and evaluate the effect of stability of the method.

\section{ACKNOWLEDGMENT}

Authors would like to thank the Federal University of Juiz de Fora, CAPES, FAPEMIG, INERGE, and CNPq for all financial, technical and scientific support on this work.

\section{REFERENCES}

[1] International Electrotechinical Commission IEC 61000-4-30 "testing and measurement techniques - Power quality measurements methods" 2003.

[2] P. Stoica, H. Li, J. Li, and S. Member, "Amplitude Estimation of Sinusoidal Signals: Survey, New Results , and an Application," 338 IEEE Trans. SIGNAL Process., vol. 48, no. 2, pp. 338-352, 2000.

[3] A. Testa, D. Gallo, R. Langella, "On the Processing of Harmonics and Interharmonics: Using Hanning Window in Standard Framework," IEEE Transactions On Power Delivery, vol. 19, no. 1, 2004.

[4] G. Benmouyal, "An Adaptive Sampling-Interval Generator for Digital Relaying,” IEEE Power Eng. Rev., vol. 9, no. 7, pp. 45-46, 1989.

[5] D. Hart, N. Damir, Y. Hu, B. Smith, and M. Egolf, "A New Frequency Tracking and Phasor Estimation Algorithm for Generator Protection," IEEE Trans. Power Deliv. Vol. 12, No. 3, July 1997, vol. 12, no. 3, pp. 1064-1073, 1997.

[6] D. Borkowski, A. Bien, "Improvement of Accuracy of Power System Spectral Analysis by Coherent Resampling," IEEE Trans. Power Delivery, vol.24, no.3, pp.1004-1013, July 2009.

[7] C. H. Martins, L. M. Silva, C. Duque, A. S. Cerqueira, E. C. Teixeira, P. F. Ribeiro, "A New Time-Varying Harmonic Decomposition Sctructure Based on Recursive Hanning Window," IEEE 15th International Conference on Harmonics and Quality of Power, 2012.

[8] J. Barros, R. I. Diego, "On the Use of the Hanning Window for Harmonic Analysis in the Standard Framework," IEEE Transactions on Power Delivery, vol.21, no.1, 2006.

[9] Z. Liu, J. Himmel, K. W. Bonfig, "Improved processing of harmonics and interharmonics by time-domain averaging," Power Delivery, IEEE Transactions on, vol.20, no.4, pp.2370-2380, Oct. 2005

[10] G. W. Chang, Cheng-I Chen, "Measurement techniques for stationary and time-varying harmonics," Power and Energy Society General Meeting, 2010 IEEE, vol., no., pp.1-5, 25-29 July 2010

[11] J. Valenzuela, J. Pontt, "Real-time interharmonics detection and measurement based on FFT algorithm," Applied Electronics, pp.259264, 9-10 Sept. 2009

[12] D. Gallo, R. Langella, A. Testa, "On the processing of harmonics and interharmonics in electrical power systems," Power Engineering Society Winter Meeting, IEEE, vol.3, pp.1581-1586 vol.3, 23-27 Jan 2000.

[13] S. Reza, M. Ciobotaru, and V. G. Agelidis, "A Recursive DFT Based Technique for Accurate Estimation of Grid Voltage Frequency," Ind. Electron. Soc., pp. 6420-6425, 2013. 